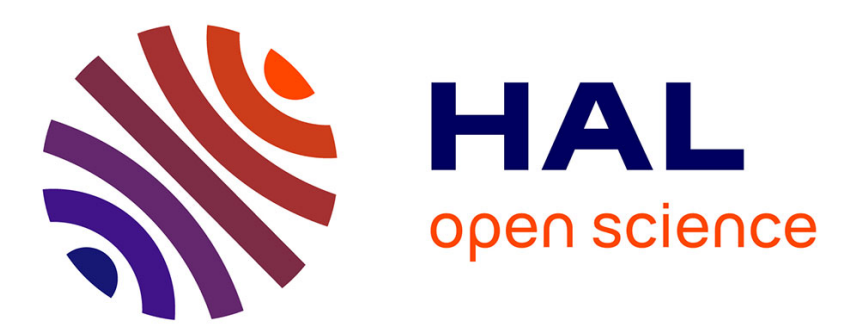

\title{
Le suivi gynécologique des femmes ayant des rapports sexuels avec des femmes: déterminants, enjeux, perspectives
}

\author{
C. Giles
}

\section{- To cite this version:}

C. Giles. Le suivi gynécologique des femmes ayant des rapports sexuels avec des femmes: déterminants, enjeux, perspectives. La Revue Sage-Femme, 2019, 18, pp.145 - 149. 10.1016/j.sagf.2019.03.002 . hal-03487035

\section{HAL Id: hal-03487035 \\ https://hal.science/hal-03487035}

Submitted on 20 Dec 2021

HAL is a multi-disciplinary open access archive for the deposit and dissemination of scientific research documents, whether they are published or not. The documents may come from teaching and research institutions in France or abroad, or from public or private research centers.
L'archive ouverte pluridisciplinaire HAL, est destinée au dépôt et à la diffusion de documents scientifiques de niveau recherche, publiés ou non, émanant des établissements d'enseignement et de recherche français ou étrangers, des laboratoires publics ou privés.

\section{다)(1) $\$$}

Distributed under a Creative Commons Attribution - NonCommerciall 4.0 International 
Le suivi gynécologique des femmes ayant des rapports sexuels avec des femmes : déterminants, enjeux, perspectives

Women who have sex with women (WSW) : their healthcare experience in gynaecology

Cécilia Giles, sage-femme

Adresse e-mail : giles_cecilia@yahoo.fr 


\section{RESUME}

Objectif. La santé sexuelle et reproductive des femmes ayant des rapports sexuels avec des femmes n'est pas considérée en tant que telle par la politique de santé publique en France. Pourtant, les études internationales mettent à jour des vulnérabilités spécifiques, en matière d'infections sexuellement transmissibles et de violences notamment. Le fait d'être une femme ayant des rapports homosexuels est associé à des disparités en matière de suivi gynécologique. La perception d'une absence de risques liés à la sexualité, la présomption d'hétérosexualité par le/la professionnel(le) ou la crainte (vécue ou anticipée) d'être mal jugée sont décrites comme des facteurs influençant négativement le recours aux soins. L'objectif de cette étude est d'explorer les parcours de santé gynécologique des femmes et de comprendre leur expérience des consultations.

Méthode. Vingt-six entretiens semi-directifs ont été menés auprès de femmes ayant des rapports sexuels avec une ou plusieurs femme(s), entre octobre 2017 et janvier 2018.

Résultats. Notre analyse montre que le manque de (re)connaissance de la sexualité entre femmes nourrit un impensé du corps gynécologique qui façonne les expériences de santé. En l'absence d'un « modèle social » à consulter, l'inscription dans un suivi gynécologique et la continuité de celui-ci sont fortement dépendantes des trajectoires individuelles (hétérosexualité, maternité, symptomatologie gynécologique).

Conclusion. L'enjeu pour le/la praticien(ne) est de prendre conscience de ses propres préjugés et de la manière dont les spécificités des patientes peuvent conduire à des inégalités de santé. Proposer une approche centrée sur la personne, 
prenant en compte les différences individuelles, sera profitable à toutes les femmes consultant en gynécologie.

Mots-clés : consultation gynécologique ; lesbienne ; femme ayant des rapports sexuels avec des femmes ; inégalités de santé.

Objective. Sexual and reproductive health of women who have sex with women (WSW) is not targeted by public health policies in France. However, international research shows that they are prone to get sexually transmitted infections and experience violence. Being a woman who have sex with women is related to a lower utilisation of gynaecological healthcare and screening programs like cervical cytology tests. The objective of this study is to explore WSW's healthcare experience in gynaecology, focusing on aspects specifically related to sexual orientation.

Methods. Twenty-six interviews were conducted with women having sex with women, from October 2017 to January 2018.

Results. Our results show that the lack of knowledge and recognition about sexuality between women influence healthcare experiences. With no existing role model about seeing a gynaecologist, health history is closely related to individual factors (experience of heterosexuality, pregnancy and gynaecological symptoms).

Conclusion. The point is to reflect upon our bias and the way women's specificities may foster health inequalities. We suggest adopting a professional attitude that is patient-centered, taking into account individual differences. This would benefit to all women consulting in gynaecology. 
Keywords : gynaecological consultation ; lesbian ; women who have sex with women ; health inequalities. 


\section{MANUSCRIT}

\section{$\underline{\text { Introduction }}$}

Tandis que les hommes gays font largement l'objet de campagnes de prévention et de dispositifs spécifiques de santé depuis l'apparition de l'épidémie de VIH/sida, les femmes ayant des rapports sexuels avec des femmes sont absentes des discours de prévention (1). Très peu d'études ont été publiées en France en ce qui concerne leur santé, notamment leur santé sexuelle et reproductive. La littérature existante provient essentiellement des pays anglosaxons et de la Suisse. Elle révèle des risques accrus d'atteinte à la santé globale de ces femmes (troubles dépressifs, addictions, surpoids, violences...), un moindre recours aux soins et en particulier à la consultation gynécologique, un moindre dépistage des infections sexuellement transmissibles (IST) - alors que celles-ci sont plus fréquemment retrouvées chez les femmes ayant des rapports homosexuels que chez les femmes hétérosexuelles - et une difficulté à dévoiler leur orientation sexuelle aux professionnel(le)s de santé ainsi que l'expérience de discriminations ou de prises en charge inadaptées.

Dans l'Enquête Presse Gays et Lesbiennes (EPGL) réalisée par l'Institut de veille sanitaire (InVS) en 2011, les femmes n'ayant eu des rapports qu'avec des femmes dans les 12 derniers mois étaient $50 \%$ à avoir eu un frottis de dépistage dans les trois dernières années contre $60 \%$ pour celles ayant eu des rapports avec des femmes et des hommes et $65 \%$ pour celles n'ayant eu que des partenaires hommes (2). Le fait que les femmes ayant des rapports sexuels avec des femmes entrent généralement plus tôt dans la vie sexuelle et qu'elles aient plus de partenaires 
sexuels (y compris masculins) que les autres femmes accroit leur susceptibilité de contracter une infection sexuellement transmissible. Ainsi, les données de l'Enquête nationale sur les violences faites aux femmes (ENVEFF) révèlent que la fréquence d'IST au cours de la vie était significativement plus élevée pour les femmes ayant eu des rapports homosexuels $(25 \%$ versus $9 \%$ pour les femmes exclusivement hétérosexuelles) (3). De même, dans l'enquête Contexte de la sexualité en France (CSF), les femmes déclarant des pratiques homosexuelles étaient $12 \%$ versus $3 \%$ pour les femmes hétérosexuelles à rapporter avoir eu une infection sexuellement transmissible dans les cinq dernières années (4).

Les femmes ayant des rapports sexuels avec des femmes présentent des facteurs de risque reconnus dans l'acquisition et la persistance de l'infection par le papillomavirus humain (HPV) : elles ont en moyenne leur premier rapport sexuel à un âge plus précoce que les femmes hétérosexuelles - 17,3 ans versus 18,6 ans (4), un

plus grand nombre de partenaires sexuels au cours de leur vie - en moyenne 14 partenaires masculins versus 4 pour les femmes hétérosexuelles (4) et une pratique tabagique plus fréquente (5-7). Par ailleurs, le papillomavirus humain peut se transmettre sexuellement entre femmes. Une étude a ainsi montré la présence de HPV chez $6 \%$ de femmes qui n'avaient eu que des partenaires féminines (8). D’autres auteurs ont également montré la présence de lésions pré-cancéreuses du col de l'utérus dans cette population $(9,10)$.

\section{Méthode}

Nous avons souhaité interroger l'expérience des femmes ayant des rapports sexuels avec des femmes en matière de suivi gynécologique, afin d'identifier dans quelle 
mesure l'orientation sexuelle est un élément important à considérer en consultation et les axes d'évolution possibles pour la pratique professionnelle. Nos objectifs de recherche étaient de comprendre comment les femmes ayant des rapports sexuels avec des femmes négocient les normes de la consultation gynécologique, d'analyser le rôle des trajectoires individuelles, affectives et sexuelles, dans l'expérience du suivi gynécologique de ces femmes ainsi que d'analyser les effets des politiques de santé sexuelle et reproductive sur une autre catégorie de femmes que celles appartenant à la catégorie majoritaire.

Nous avons construit notre grille d'entretien sur une approche holistique des personnes, abordant les représentations de la santé, les expériences en matière de sexualité, le rapport aux autres et le vécu de l'homosexualité, pour mieux appréhender le parcours gynécologique des femmes. Le recrutement s'est décliné en deux phases : un premier appel à participation a été lancé par le biais du réseau social Facebook, de la liste de diffusion féministe Efigies et de notre réseau personnel ; un deuxième appel à participation s'est orienté vers le réseau associatif. Au total, nous avons réalisé 26 entretiens semi-directifs, d'une durée moyenne d'une heure, entre les mois d'octobre 2017 et janvier 2018. L'intégralité des entretiens a été retranscrite pour l'analyse.

Les participantes de notre étude sont âgées de 21 à 59 ans. Dix-sept femmes ont moins de 35 ans et neuf femmes ont plus de 35 ans (21-29 ans : 13 femmes ; 30-39 ans : 5 femmes ; 40-49 ans : 4 femmes ; 50-59 ans : 4 femmes). Leur profil est semblable à celui des femmes ayant déclaré des rapports homosexuels dans l'enquête ENVEFF et dans les enquêtes menées dans d'autres pays sur les 
comportements sexuels ou la santé. Elles vivent très majoritairement dans les grandes agglomérations et notamment la région parisienne. Elles ont un niveau d'études élevé et sont en emploi. Elles sont majoritairement en union non officialisée par un mariage ou un pacte civil de solidarité (PACS) et plus souvent en couple non cohabitant que les femmes hétérosexuelles.

Les femmes de notre étude relèvent pour six d'entre elles d'un parcours sexuel exclusif (elles n'ont jamais eu de relations sexuelles avec des hommes), pour huit d'un parcours sexuel simultané (elles alternent ou ont alterné des relations avec des femmes et avec des hommes) et pour douze d'un parcours sexuel progressif (elles ont eu des relations engagées affectivement avec des hommes ; le plus souvent, la période hétérosexuelle a précédé la période homosexuelle) (11).

\section{Résultats}

La santé gynécologique des femmes ayant des rapports sexuels avec des femmes est un sujet non problématisé

Un sentiment d'immunité sexuelle a été décrit par une grande majorité des femmes enquêtées. Leurs rapports sexuels entre femmes ne sont pas protégés et elles ont très peu recours au dépistage des IST. Elles associent la consultation gynécologique à la contraception et aux risques hétérosexuels. 
Cette perception d'une absence de risque se trouve renforcée par une absence d'information et de guidance des professionnel(le)s de santé et des politiques publiques, qui ne portent pas de discours sur le parcours de santé gynécologique d'une femme ayant des rapports sexuels avec des femmes. Pourtant, trois femmes de notre étude (sur 26) ont été concernées par une infection à papillomavirus humain (HPV) : une femme a été traitée par laser pour des condylomes et deux femmes ont eu une conisation suite à la découverte d'une lésion de haut grade au frottis. Toutes avaient eu des rapports sexuels avec des hommes. Pour l'une d'elle, au moment du dépistage, cela faisait près de vingt ans qu'elle n'avait pas eu de relations hétérosexuelles.

Cet état de fait expose les femmes ayant des rapports sexuels avec des femmes aux inégalités sociales de santé, puisque le savoir concernant la prévention et la santé gynécologique reste confiné aux espaces militants et donc accessible aux femmes les plus insérées dans le milieu lesbien. Les enquêtées issues du recrutement par le biais des associations sont apparues les mieux informées des risques et des moyens de prévention des IST (digue dentaire, gants, préservatifs pour les objets sexuels).

\section{L'initiation du parcours gynécologique est déterminée par trois types d'expérience}

II est apparu au cours des entretiens que, contrairement aux jeunes femmes hétérosexuelles, celles dont l'homosexualité est connue des parents ne sont pas incitées à consulter en gynécologie. L'initiation du parcours gynécologique est donc 
essentiellement déterminée par trois types d'expérience personnelle: l'hétérosexualité, la maternité et les plaintes somatiques.

L'hétérosexualité influence souvent la précocité de la première consultation. C'est une expérience largement partagée, comme le montre la prédominance des parcours sexuels simultanés et progressifs parmi nos enquêtées. Le vécu de cette expérience est souvent relié au poids des normes familiales et sociétales.

La maternité est apparue comme un événement fondateur pour plusieurs femmes qui n'avaient jamais ou quasiment jamais consulté (relevant d'un parcours sexuel exclusif). Ce fut l'occasion pour elles de créer un lien avec le milieu médical et un effet « collatéral » sur la compagne a été rapporté. Les enquêtées ont exprimé un apaisement voire un soulagement dans leur rapport au suivi gynécologique.

Les plaintes somatiques, essentiellement les dysménorrhées et les mycoses, ont été identifiées comme un élément déterminant de la démarche de consultation, notamment chez les femmes ayant un parcours sexuel exclusif. Le médecin généraliste a été cité à plusieurs reprises comme un interlocuteur privilégié sur ces sujets.

L'analyse des parcours gynécologiques des participantes a permis d'identifier des facteurs de risque de discontinuité voire de rupture du parcours gynécologique ainsi que des facteurs protecteurs 
Les facteurs de risque identifiés sont:

- l'absence de « modèle social à consulter » chez les femmes ayant des rapports sexuels avec des femmes : elles échangent peu avec leur(s) partenaire(s) et amies homosexuelles, celles-ci ne consultant pas ou peu elles-mêmes.

- une moindre estime de soi, avec des troubles dépressifs exprimés par plusieurs participantes (à relier avec la littérature existante).

- des problématiques spécifiques dans le rapport au corps et à la consultation gynécologique : des enquêtées ont exprimé leur difficulté à appréhender des parties du corps symboliques de la féminité telles que les seins, ou la pénibilité d'un examen gynécologique vécu comme une mise en scène sexualisée du corps (sexualité digitale).

- de mauvaises expériences en consultation : il a été rapporté des marques d'incompréhension, de malaise voire de jugement de la part du/de la professionnel(le) de santé suite au dévoilement de l'orientation sexuelle ; pourtant les femmes considèrent généralement pertinent de le dire au/à la soignant(e) pour être correctement prises en charge.

- le changement d'orientation sexuelle : la majorité de participantes dont le parcours sexuel est progressif ont rapporté une discontinuité voire une rupture dans leur suivi gynécologique. Certaines sont convaincues qu'il n'y a plus d'intérêt à consulter tandis que d'autres s'interrogent sur la nécessité de continuer à consulter ou non, et à quelle fréquence.

Parmi les facteurs protecteurs du suivi, on retrouve : 
- la sensibilisation par la famille, notamment lorsqu'il y a des cas de cancer du sein dans la famille, qui joue un rôle bénéfique y compris en cas de mauvaises expériences de consultation.

- le fait de trouver « le/la bon(ne) praticien(ne) »: les femmes ont décrit un phénomène de recommandation de praticiens dits "safe ", dans le milieu lesbien ou par le biais de listes de soignant(e)s référencé(e)s par les patientes elles-mêmes.

\section{Synthèse}

L'absence de discours préventif à l'intention des femmes ayant des rapports sexuels avec des femmes nourrit leur sentiment d'une absence de risques et leur moindre recours aux pratiques de protection et aux dépistages. Nous avons constaté que cet impensé du corps gynécologique se trouve renforcé par un défaut d'information et de guidance par les professionnel(le)s de santé ; les manques de formation et de réflexion autour de la déconstruction des rôles socio-sexuels et des stéréotypes de genre apparaissent être les principales causes.

Cet état de fait expose les femmes ayant des rapports homosexuels de manière accrue aux inégalités sociales de santé. En effet, celles qui sont les plus insérées dans le réseau lesbien bénéficient d'une information et d'un soutien qui n'ont pas d'équivalents par ailleurs. Les jeunes femmes dont l'homosexualité est connue des parents ne sont pas incitées, pour la plupart, à consulter en gynécologie. Notre analyse montre que ce sont les expériences de l'hétérosexualité, de la maternité ou 
l'apparition d'une symptomatologie gynécologique qui sont généralement les prescriptrices de la démarche de consulter. L'inscription dans un suivi gynécologique et la continuité de celui-ci sont donc fortement dépendantes des trajectoires individuelles.

Enfin, il apparaît singulièrement une absence de « modèle social » à consulter en gynécologie parmi les femmes lesbiennes. La centralité de la norme contraceptive ainsi que la difficulté à trouver un espace pour dialoguer ouvertement de son orientation sexuelle et de ses besoins pourraient l'expliquer. La méconnaissance ou l'absence de considération pour la sexualité entre femmes conduit une grande partie des femmes ayant des rapports sexuels avec des femmes à ne pas appréhender la consultation gynécologique comme un espace ressource pour leur santé. Elles hésitent à dévoiler leur orientation sexuelle, ce qui renforce la présomption d'hétérosexualité largement répandue chez les professionnel(le)s et peut résulter en une offre de santé inadaptée.

\section{$\underline{\text { Conclusion et recommandations pour la pratique professionnelle }}$}

Plusieurs études internationales sur l'interaction soignant(e)/soignée pour les femmes ayant des rapports sexuels avec des femmes ont montré que lorsque le médecin est informé de l'orientation sexuelle de sa patiente, cela peut avoir des impacts positifs sur le recours aux soins $(12,13)$. II n'est pas question de préconiser une approche particulière pour les femmes ayant des rapports sexuels avec des femmes, mais plutôt une approche centrée sur la personne prenant en compte les 
différences individuelles. Les enjeux sont avant tout d'offrir un environnement accueillant et sécurisant et d'aborder sans a priori et de manière non jugeante les questions de sexualité, d'orientation sexuelle et d'identité de genre. Le dialogue permet d'adapter les conseils de prévention, les dépistages et les éventuels traitements aux pratiques réelles, puisque celles-ci ne coïncident pas nécessairement avec l'orientation sexuelle déclarée ou supposée. Devant des pratiques inconnues que la patiente peut révéler, le questionnement de la part du/de la professionnel(le) peut être pris comme une marque d'intérêt s'il est empathique. Le fait de prendre conscience de ses propres préjugés et de la manière dont les spécificités des patientes peuvent conduire à des inégalités de santé nous paraît essentiel. Il est important de proposer l'examen gynécologique (à adapter à chacune), les dépistages des cancers du col de l'utérus et du sein (pertinents pour toutes les femmes) et la prescription de la pilule à d'autres fins que la contraception (notamment pour améliorer des dysménorrhées).

Le sujet des violences doit être abordé, car il n'est pas exempt des relations entre femmes. De plus, des études ont montré l'incidence élevée des violences chez les femmes ayant des rapports sexuels avec des femmes en raison de leur parcours biographique, marqué par une entrée plus précoce dans la sexualité et un plus grand nombre de partenaires, y compris masculins.

Enfin, il est établi que le bien-être et l'estime de soi sont déterminants dans la capacité d'un individu à se projeter dans une démarche de soin et de prévention. La stigmatisation ou les discriminations, qu'elles soient institutionnelles ou individuelles, vécues par les femmes ayant des rapports sexuels avec des femmes entraînent 
fréquemment des problèmes d'estime de soi, d'anxiété, ainsi qu'une incidence plus élevée de troubles dépressifs et de comportements suicidaires. Cela est particulièrement retrouvé chez la population adolescente, qui doit faire face au trouble et à la recherche identitaire propre à cette période de la vie, en même temps qu'affronter les premiers questionnements sur l'orientation sexuelle et gérer l'annonce de l'homo/bisexualité à l'entourage (14). Le malaise psychologique serait également un peu plus fréquent chez les femmes dont l'homosexualité n'est pas agie, c'est-à-dire qui sont attirées par des femmes mais non engagées dans des pratiques homosexuelles (15).

Les sages-femmes sont directement concernées par le sujet. Leurs compétences en matière de suivi gynécologique de prévention en font des praticiennes de premiers recours pour la majorité des femmes. De la même façon, ce n'est pas parce que l'aide médicale à la procréation n'est pas (encore) autorisée en France pour les couples de femmes que celles-ci renoncent à leur projet de fonder une famille. L'expérience de la maternité s'est révélée structurante pour les femmes que nous avons interrogées et représente une occasion privilégiée pour les professionnel(le)s de créer du lien, autant avec la patiente qu'avec sa partenaire. 


\section{REMERCIEMENTS}

Nous remercions Hélène Malmanche, sage-femme et doctorante en sociologie, pour son enthousiasme et ses précieux conseils dans la direction de ce travail ; celles et ceux qui ont permis la rencontre avec les participantes de l'étude, en diffusant notre appel à participation dans leurs réseaux professionnel et personnel ; les femmes qui ont accepté de partager avec nous leur expérience des consultations gynécologiques, elles se sont livrées intimement et ont nourri la richesse de cette recherche. 


\section{DECLARATION DE LIENS D'INTERETS}

L'auteure déclare ne pas avoir de liens d'intérêts. 


\section{REFERENCES}

(1) Genon C, Chartrain C, Delebarre C. Pour une promotion de la santé lesbienne $\square$ : état des lieux des recherches, enjeux et propositions. Genre Sex Société [Internet]. Juin 2009 [consulté le 12 mai 2018];(1). Disponible sur: http://journals.openedition.org/gss/951

(2) Velter A, Bouyssou A, Saboni L, Bernillon P, Sommen C, Methy N. Enquête Presse Gays et Lesbiennes (EPGL) 2011. InVS, ANRS; 2011.

(3) Saurel-Cubizolles M-J, Lhomond B. Les femmes qui ont des relations homosexuelles $\square$ : leur biographie sexuelle, leur santé reproductive et leur expérience des violences. Gynécologie Obstétrique Fertil. 2005;Vol. 33(10):776ロ82.

(4) Bajos N, Bozon M. Enquête sur la sexualité en France. Pratiques, genre et santé. La Découverte. Paris; 2008. 612 p. (Hors Collection Social).

(5) Tang H, Greenwood GL, Cowling DW, Lloyd JC, Roeseler AG, Bal DG. Cigarette Smoking Among Lesbians, Gays, and Bisexuals: How Serious a Problem? (United States). Cancer Causes Control. Oct 2004;15(8):797ロ 803.

(6) Boehmer U, Miao X, Linkletter C, Clark MA. Adult health behaviors over the life course by sexual orientation. Am J Public Health. Févr 2012;102(2):292 $\square 300$.

(7) Johnson SE, Holder-Hayes E, Tessman GK, King BA, Alexander T, Zhao X. Tobacco Product Use Among Sexual Minority Adults: Findings From the 2012-2013 National Adult Tobacco Survey. Am J Prev Med. Avr 2016;50(4):e91 $\square 100$.

(8) Marrazzo JM, Koutsky LA, Kiviat NB, Kuypers JM, Stine K. Papanicolaou test screening and prevalence of genital human papillomavirus among women who have sex with women. Am J Public Health. Juin 2001;91(6):947 $\square 52$. 
(9) Bailey JV, Kavanagh J, Owen C, McLean KA, Skinner CJ. Lesbians and cervical screening. Br J Gen Pr. Juin 2000;50(455):481 $\square 2$.

(10) Massad LS, Xie X, Minkoff H, Darragh TM, D'Souza G, Sanchez-Keeland L, et al. Abnormal Pap tests and human papillomavirus infections among HIV infected and uninfected women who have sex with women. J Low Genit Tract Dis. Janv 2014;18(1):50ロ6.

(11) Chetcuti N. Se dire lesbienne. Vie de couple, sexualité, représentation de soi. Payot. 2010. 300 p.

(12) Steele LS, Tinmouth JM, Lu A. Regular health care use by lesbians: a path analysis of predictive factors. Fam Pract. Déc 2006;23(6):631 $\square 6$.

(13) Tracy JK, Schluterman NH, Greenberg DR. Understanding cervical cancer screening among lesbians: a national survey. BMC Public Health. Mai 2013;13:442.

(14) Bozon M. Sociologie de la sexualité. Nathan. Paris; 2002. 128 p.

(15) Lhomond B, Saurel-Cubizolles M-J. Orientation sexuelle, violences contre les femmes et santé $\square$ : résultats de l'enquête nationale sur les violences envers les femmes en France. In: Homosexualités au temps du sida $\square$ : tensions sociales et identitaires [Internet]. Agence nationale de recherche sur le sida. Paris; 2003 [consulté le 12 mai 2018]. (Collection Sciences sociales et sida). Disponible sur: http://semgai.free.fr/doc_et_pdf/homo_anrs_crips2004.pdf 